\title{
TIMASSS: the IRAS16293-2422 millimeter and submillimeter spectral survey: tentative detection of deuterated methyl formate $\left(\mathrm{DCOOCH}_{3}\right)^{\star, \star \star}$
}

\author{
K. Demyk ${ }^{1,2}$, S. Bottinelli1 ${ }^{1,2}$, E. Caux ${ }^{1,2}$, C. Vastel ${ }^{1,2}$, C. Ceccarelli ${ }^{3}$, C. Kahane ${ }^{3}$, and A. Castets ${ }^{3}$ \\ ${ }^{1}$ CESR (Centre d'étude Spatiale des Rayonnements), Université de Toulouse [UPS], 31028 Toulouse Cedex 4, France \\ e-mail: karine.demyk@cesr.fr \\ 2 CNRS, UMR 5187, 9 avenue du Colonel Roche, 31028 Toulouse Cedex 4, France \\ Laboratoire d'Astrophysique, Observatoire de Grenoble, BP 53, 38041 Grenoble Cedex 9, France
}

Received 9 December 2009 / Accepted 14 April 2010

ABSTRACT

\begin{abstract}
Context. High deuterium fractionation is observed in various types of environments such as prestellar cores, hot cores, and hot corinos. It has proven to be an efficient probe for studying the physical and chemical conditions of these environments. The study of the deuteration of different molecules helps us to understand their formation. This is especially interesting for complex molecules like methanol and bigger molecules for which it may allow differentiation of gas-phase and solid-state formation pathways.

Aims. Methanol exhibits a high deuterium fractionation in hot corinos. Since $\mathrm{CH}_{3} \mathrm{OH}$ is thought to be a precursor of methyl formate we expect that deuterated methyl formate is produced in such environments. We searched for the singly-deuterated isotopologue of methyl formate, $\mathrm{DCOOCH}_{3}$, in IRAS 16293-2422, a hot corino well-known for its high degree of methanol deuteration.

Methods. We used the IRAM/JCMT unbiased spectral survey of IRAS 16293-2422, which allowed us to search for the $\mathrm{DCOOCH}_{3}$ rotational transitions within the survey spectral range $(80-280 \mathrm{GHz}, 328-366 \mathrm{GHz})$. The expected emission of deuterated methyl formate is modelled at LTE and compared with the observations.

Results. We have tentatively detected $\mathrm{DCOOCH}_{3}$ in the protostar IRAS 16293-2422. We assign eight lines detected in the IRAM survey to DCOOCH3. Three of these lines are affected by blending problems, and one is affected by calibration uncertainties; nevertheless, the LTE emission model is compatible with the observations. A simple LTE modelling of the two cores in IRAS 16293-2422, based on a previous interferometric study of $\mathrm{HCOOCH}_{3}$, allows us to estimate the amount of DCOOCH $\mathrm{D}_{3}$ in IRAS 16293-2422. Adopting an excitation temperature of $100 \mathrm{~K}$ and a source size of $2^{\prime \prime}$ and 1.5 for the A and B cores, respectively, we find that $N_{\mathrm{A}, \mathrm{DCOOCH} 3}=N_{\mathrm{B}, \mathrm{DCOOCH} 3} \sim 6 \times 10^{14} \mathrm{~cm}^{-2}$. The derived deuterium fractionation is $\sim 15 \%$, consistent with values for other deuterated species in this source and much greater than expected from the deuterium cosmic abundance.

Conclusions. If its tentative detection is confirmed, $\mathrm{DCOOCH}_{3}$ should now be considered in theoretical models that study complex molecule formation and their deuteration mechanisms. Experimental work is also needed to investigate the different chemical routes leading to the formation of deuterated methyl formate.
\end{abstract}

Key words. line: identification - methods: observational - ISM: molecules - ISM: abundances -

ISM: individual objects: IRAS 16293-2422 - radio lines: ISM

\section{Introduction}

IRAS 16293-2422 (hereafter IRAS 16293) is a complex source hosting two hot corinos (called "A" and "B") in which many complex organic molecules have been observed: acetonitrile $\left(\mathrm{CH}_{3} \mathrm{CN}\right)$, methyl formate $\left(\mathrm{HCOOCH}_{3}\right)$, ketene $\left(\mathrm{H}_{2} \mathrm{CCO}\right)$, formic acid $(\mathrm{HCOOH})$, ethanol $\left(\mathrm{C}_{2} \mathrm{H}_{5} \mathrm{OH}\right)$, ethyl cyanide $\left(\mathrm{C}_{2} \mathrm{H}_{5} \mathrm{CN}\right)$, etc. (Cazaux et al. 2003; Bottinelli et al. 2004; Bisschop et al. 2008). This source is also characterised by a high degree of deuteration with singly, doubly, or triply deuterated molecules. $\mathrm{HDCO}, \mathrm{CH}_{3} \mathrm{OD}$, and $\mathrm{CH}_{2} \mathrm{DOH}$ are all detected

* Based on observations carried out with the IRAM 30 m telescope. IRAM is supported by INSU/CNRS (France), MPG (Germany) and IGN (Spain).

$\star \star$ Based on analysis carried out with the CASSIS software and the JPL (http://spec.jpl.nasa.gov/) and CDMS (http://www.ph1.uni-koeln.de/cdms/) spectroscopic databases. CASSIS has been developed by CESR-UPS/CNRS (http://cassis.cesr.fr). in IRAS 16293 with a deuterium fractionation of $\sim 15 \%, 1.8 \%$ and $37 \%$, respectively, while doubly-deuterated formaldehyde $\left(\mathrm{D}_{2} \mathrm{CO}\right)$ and methanol $\left(\mathrm{CHD}_{2} \mathrm{OH}\right)$ are observed with a deuteration of 5 and $7.4 \%$ (Parise et al. 2006). Triply deuterated methanol was also observed in IRAS 16293 with a deuterium fractionation ratio of 1.4\% (Parise et al. 2004).

These values are much greater than expected from the cosmic $\mathrm{D} / \mathrm{H}$ ratio $\left(\sim 10^{-5}\right)$. The process of deuteration is a longstanding and challenging issue. High deuterium fractionation is observed in cold regions such as pre-stellar cores (Pillai et al. 2007; Bacmann et al. 2003), in warmer regions such as hot cores (eg. Orion, Jacq et al. 1993) and hot corinos and in photo dissociation regions (PDRs) (Leurini et al. 2006; Pety et al. 2007). The detection of deuterated molecules allows one to study the chemical pathways leading to their formation and to trace the chemical and physical conditions of the observed environments. Whereas the deuterium molecular enrichment is explained by gas phase chemistry in cold environments (Roberts et al. 2003), in hot regions it is certainly driven by chemistry on the icy surface of 
the dust (Tielens 1983; Ceccarelli et al. 2001). This is illustrated well by the detection of multiply deuterated methanol since methanol cannot be efficiently formed in the gas phase (Turner 1998). Since methanol is thought to be involved in methyl formate formation on grains (Bennett \& Kaiser 2007), the high deuterium fractionation of $\mathrm{CH}_{3} \mathrm{OH}$ suggests that deuterated methyl formate should be produced along with methyl formate.

In this study we present the tentative detection of deuterated methyl formate, $\mathrm{DCOOCH}_{3}$, in IRAS 16293 based on observations from the millimeter/submillimeter spectral survey performed toward the low-mass Class 0 protostar IRAS16293 (Caux et al. 2010). The observations are described in Sect. 2. The tentative detection of $\mathrm{DCOOCH}_{3}$ is presented in Sect. 3 and its formation is discussed in Sect. 4.

\section{Observations}

The survey was carried out with the IRAM-30m (frequency range $80-280 \mathrm{GHz}$ ) and JCMT-15m (frequency range 328$366 \mathrm{GHz}$ ) telescopes during the period January 2004 to August 2006. The angular resolution (HPBW) of the observations varied between $9^{\prime \prime}$ and $33^{\prime \prime}$, depending on the telescope and frequency used, and the spectral resolution ranged between 0.3 and 1.2 MHz. All observations were performed in double-beamswitch observing mode, with a $90^{\prime \prime}$ throw. Pointing and focus were regularly checked, and the resulting pointing accuracy depended on the weather and the observed frequency but was always better than $5^{\prime \prime}$. The lines assigned to deuterated methyl formate presented in this paper were observed in the $190-270 \mathrm{GHz}$ domain at the IRAM $30 \mathrm{~m}$, with a spectral resolution of $1-$ $1.25 \mathrm{MHz}$ and an rms of about 5-15 mK (antenna temperature). For a detailed description of this survey, see the paper presenting the observations and calibrations of the whole survey (Caux et al. 2010). Once this paper has been published, the data will be made publicly available on the TIMASSS web site (http: // www-laog.obs.ujf-grenoble. fr/heberges/timasss/).

\section{Results}

To search for deuterated methyl formate, we used the predicted spectrum calculated from the recent study by Margulès et al. (2010). Deuterated methyl formate $\mathrm{DCOOCH}_{3}$ is an asymmetric molecule described by the quantum numbers $J, K_{\mathrm{a}}$, and $K_{\mathrm{c}}$. It has a dense rotational spectrum in which each transition is split into $\mathrm{A}$ and $\mathrm{E}$ components of the same intensity because of the internal rotation of the methyl group.

Figure 1 shows the emission spectrum of $\mathrm{DCOOCH}_{3}$ modelled with the CASSIS software ${ }^{1}$ in LTE, assuming optically thin conditions, with an excitation temperature of $100 \mathrm{~K}$ (typical of hot core values), a line width of $5 \mathrm{~km} \mathrm{~s}^{-1}$, and an extended source $\left(100^{\prime \prime}\right)$. The aim of this figure is to investigate the intensity ratios of the $\mathrm{DCOOCH}_{3}$ transitions, and it can indeed be seen that some lines are strikingly more intense than the others. Each of these strong lines consists of 8 transitions with $J^{\prime \prime} \geq 18$ and $K_{\mathrm{a}}^{\prime}-K_{\mathrm{a}}^{\prime \prime}=0-1,0-0,1-0$, and $1-1$ (4 for the A-species and 4 for the E-species, see Table 1) which are very close in frequency and are blended in the modelled spectrum because of the line width.

Considering that the line width of complex molecules in IRAS 16293 is a few $\mathrm{km} \mathrm{s}^{-1}\left(2-6 \mathrm{~km} \mathrm{~s}^{-1}\right.$ for $\mathrm{HCOOCH}_{3}$, Bottinelli et al. 2004), these very intense lines are particularly

\footnotetext{
1 The CASSIS software is a free interactive spectrum analyser aiming to interpret astrophysical spectra, for more details see http:// cassis. cesr. fr
}

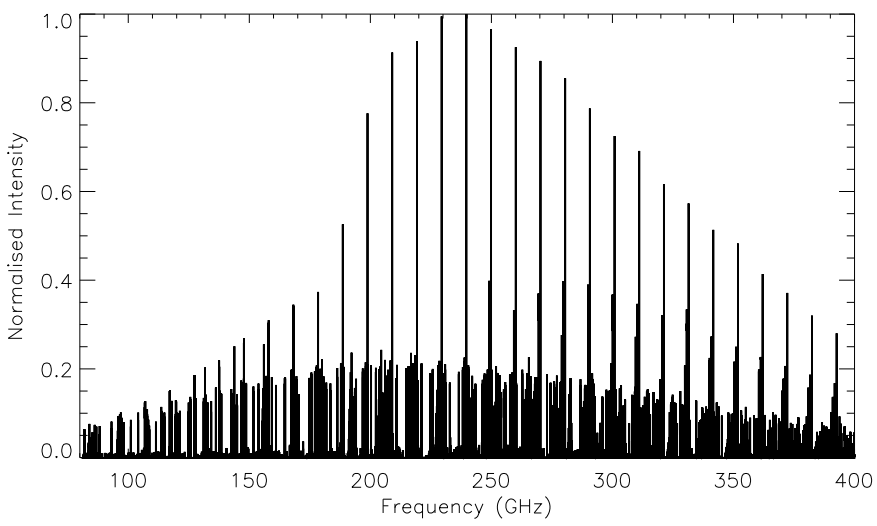

Fig. 1. LTE emission model of $\mathrm{DCOOCH}_{3}$ calculated in optically thin conditions for an extended source, an excitation temperature of $100 \mathrm{~K}$, with a linewidth of $5 \mathrm{~km} \mathrm{~s}^{-1}$. The line intensity has been normalised to the strongest line at $239.655 \mathrm{GHz}$.

favourable to search for $\mathrm{DCOOCH}_{3}$. Eleven of these lines are in the observed spectral range of the IRAS 16293 spectral survey, eight in the IRAM data, and three in the JCMT data. In the JCMT spectral range, because the modelled line intensity decreases (Fig. 1) and the rms of the observed spectrum is high ( $15-25 \mathrm{mK}$, main beam temperature), the line intensities predicted by the LTE model described below are within the noise. Consequently, we do not detect these transitions. We assigned eight lines detected in the IRAM spectral range to the other eight transitions of $\mathrm{DCOOCH}_{3}$ (Table 1). All other lines from $\mathrm{DCOOCH}_{3}$ are below the detection limit. Indeed, for the other transitions of $\mathrm{DCOOCH}_{3}$, i.e. transitions with $J^{\prime \prime} \geq 18$ and $K_{\mathrm{a}}^{\prime}>1$ and transitions with $J^{\prime \prime}<18$ and for all value of $K_{\mathrm{a}}^{\prime}$ (i.e. frequency $<190 \mathrm{GHz}$ ), the transition frequencies are more spaced out. Consequently the lines are not blended anymore, and their intensity is low (Fig. 1). At 2 and $3 \mathrm{~mm}$, this is combined with a beam dilution effect since the emission region is supposed to be compact.

To compare with the observations, we modelled the $\mathrm{DCOOCH}_{3}$ emission in LTE. Although the single-dish observations do not allow us to constrain the spatial distribution of $\mathrm{DCOOCH}_{3}$ it seems reasonable to assume that it follows that of the main isotopologue. Thus, following the interferometric observations of $\mathrm{HCOOCH}_{3}$ from Bottinelli et al. (2004) we used a two-component emission model characterised as follows: an angular size, $\theta_{\mathrm{s}}$, of $2^{\prime \prime}, V_{\mathrm{lsr}}=3.9 \mathrm{~km} \mathrm{~s}^{-1}$, and a line width $\Delta v$ of $6 \mathrm{~km} \mathrm{~s}^{-1}$ for source $\mathrm{A}$ and $\theta_{\mathrm{s}}=1^{\prime \prime} .5, V_{\mathrm{lsr}}=2.7 \mathrm{~km} \mathrm{~s}^{-1}$, and $\Delta v=2 \mathrm{~km} \mathrm{~s}^{-1}$ for source $\mathrm{B}$. The excitation temperature was set to $100 \mathrm{~K}$ for both emission regions, a value derived for $\mathrm{HCOOCH}_{3}$ in the spectral survey (see below). A comparison between the observations and the model is presented in Fig. 2.

The transition at $198.824 \mathrm{GHz}$ is potentially blended with a bright $\mathrm{SO}$ line in the image sideband $((5,4)-(4,3)$ at 206.175 GHz, observed $T_{\mathrm{A}}{ }^{*}=2.5 \mathrm{~K}$, the line shows up at $198.826 \mathrm{GHz}$ in the signal band), however, there is a problem with the calibration of the observations at this frequency. Indeed, adopting a sideband rejection of $15 \mathrm{~dB}$ (default value), we find that the SO contaminating line from the image band would have $T_{\mathrm{A}}{ }^{*} \sim 80 \mathrm{mK}$, contradicting the observed line at $198.826 \mathrm{GHz}$ $\left(T_{\mathrm{A}}{ }^{*} \sim 35 \mathrm{mK}\right)$. The assumed sideband rejection thus seems to be incorrect and without knowing the true rejection of this observation we cannot conclude whether the line is caused by SO alone or by $\mathrm{DCOOCH}_{3}$ and either SO or another unidentified species. This line should thus be considered with caution. 
K. Demyk et al.: Deuterated methyl formate $\left(\mathrm{DCOOCH}_{3}\right)$ in IRAS 16293-1422

Table 1. Emission lines assigned to $\mathrm{DCOOCH}_{3}$ in the IRAS 16293 IRAM spectral survey.

\begin{tabular}{|c|c|c|c|c|c|c|c|}
\hline $\begin{array}{c}\text { Trans. } \\
J_{K_{\mathrm{a}}^{\prime} K_{\mathrm{c}}^{\prime}}^{\prime}-J_{K_{\mathrm{a}}^{\prime \prime} K_{\mathrm{c}}^{\prime \prime}}^{\prime \prime}\end{array}$ & $\begin{array}{l}\text { Pred. } \\
\text { freq. } \\
(\mathrm{MHz})\end{array}$ & $\begin{array}{l}\mathrm{S} \mu^{2} \\
\left(\mathrm{D}^{2}\right)\end{array}$ & $\begin{array}{c}E_{\text {up }} \\
\left(\mathrm{cm}^{-1}\right)\end{array}$ & $\begin{array}{l}\text { Obs. } \\
\text { freq. }^{a} \\
(\mathrm{MHz})\end{array}$ & $\begin{array}{c}\text { Obs. } \\
\int T_{\mathrm{mb}} \delta v \\
\left(\mathrm{mK} \mathrm{km} \mathrm{s}^{-1}\right)^{b}\end{array}$ & $\begin{array}{l}\text { Uncertertainty } \\
\text { Obs. } \int T_{\mathrm{mb}} \delta v \\
\left(\mathrm{mK} \mathrm{km} \mathrm{s}^{-1}\right)^{c}\end{array}$ & $\begin{array}{c}\text { Pred. } \\
\int T_{\mathrm{mb}} \delta v \\
\left(\mathrm{mK} \mathrm{km} \mathrm{s}^{-1}\right)^{d}\end{array}$ \\
\hline $19_{0,19}-18_{1,18} \mathrm{E}$ & 198821.94 & 9.77 & \multirow{8}{*}{61} & \multirow{8}{*}{$198826.65^{*}$} & \multirow{8}{*}{289} & \multirow{8}{*}{63} & \multirow{7}{*}{258} \\
\hline $19_{0,19}-18_{1,18} \mathrm{~A}$ & 198823.01 & 9.77 & & & & & \\
\hline $19_{1,19}-18_{1,18} \mathrm{E}$ & 198823.21 & 50.85 & & & & & \\
\hline $19_{1,19}-18_{1,18} \mathrm{~A}$ & 198824.28 & 50.86 & & & & & \\
\hline $19_{0,19}-18_{0,18} \mathrm{E}$ & 198824.38 & 50.85 & & & & & \\
\hline $19_{0,19}-18_{0,18} \mathrm{~A}$ & 198825.45 & 50.86 & & & & & \\
\hline $19_{1,19}-18_{0,18} \mathrm{E}$ & 198825.65 & 9.77 & & & & & \\
\hline $19_{1,19}-18_{0,18} \mathrm{~A}$ & 198826.72 & 9.76 & & & & & \\
\hline $20_{0,20}-19_{1,19} \mathrm{E}$ & 209031.87 & 10.32 & \multirow{9}{*}{68} & \multirow{9}{*}{$209031.80^{*}$} & \multirow{9}{*}{546} & \multirow{9}{*}{59} & \multirow{9}{*}{275} \\
\hline $20_{1,20}-19_{1,19} \mathrm{E}$ & 209032.54 & 53.55 & & & & & \\
\hline $20_{0,20}-19_{1,19} \mathrm{~A}$ & 209032.89 & 10.32 & & & & & \\
\hline $20_{0,20}-19_{0,19} \mathrm{E}$ & 209033.15 & 53.55 & & & & & \\
\hline $20_{1,20}-19_{1,19} \mathrm{~A}$ & 209033.55 & 53.56 & & & & & \\
\hline $20_{1,20}-19_{0,19} \mathrm{E}$ & 209033.81 & 10.32 & & & & & \\
\hline $20_{0,20}-19_{0,19} \mathrm{~A}$ & 209034.16 & 10.32 & & & & & \\
\hline $20_{1,20}-19_{0,19} \mathrm{~A}$ & 209034.82 & 53.56 & & & & & \\
\hline $21_{0,21}-20_{1,20} \mathrm{E}$ & 219240.53 & 10.88 & & & & & \\
\hline $21_{1,21}-20_{1,20} E$ & 219240.88 & 56.26 & \multirow{7}{*}{75} & \multirow{7}{*}{$219242.75^{*}$} & \multirow{7}{*}{614} & \multirow{7}{*}{54} & \multirow{6}{*}{281} \\
\hline $21_{0,21}-20_{0,20} \mathrm{E}$ & 219241.20 & 56.26 & & & & & \\
\hline $21_{0,21}-20_{1,20} \mathrm{~A}$ & 219241.49 & 10.87 & & & & & \\
\hline $21_{1,21}-20_{0,20} \mathrm{~A}$ & 219241.54 & 10.88 & & & & & \\
\hline $21_{1,21}-20_{1,20} \mathrm{~A}$ & 219241.84 & 56.26 & & & & & \\
\hline $21_{0,21}-20_{0,20} \mathrm{~A}$ & 219242.16 & 56.26 & & & & & \\
\hline $21_{1,21}-20_{0,20} \mathrm{~A}$ & 219242.50 & 10.87 & & & & & \\
\hline $22_{0,22}-21_{1,21} \mathrm{E}$ & 229448.05 & 11.43 & \multirow{9}{*}{82} & \multirow{9}{*}{229448.88} & \multirow{9}{*}{297} & \multirow{9}{*}{89} & \multirow{9}{*}{275} \\
\hline $22_{1,22}-21_{1,21} \mathrm{E}$ & 229448.23 & 58.96 & & & & & \\
\hline $22_{0,22}-21_{0,21} E$ & 229448.40 & 58.96 & & & & & \\
\hline $22_{1,22}-21_{0,21} \mathrm{E}$ & 229448.57 & 11.43 & & & & & \\
\hline $22_{0,22}-21_{1,21} \mathrm{~A}$ & 229448.95 & 11.43 & & & & & \\
\hline $22_{1,22}-21_{1,21} \mathrm{~A}$ & 229449.13 & 58.97 & & & & & \\
\hline $22_{0,22}-21_{0,21} \mathrm{~A}$ & 229449.29 & 58.97 & & & & & \\
\hline $22_{1,22}-21_{0,21} \mathrm{~A}$ & 229449.47 & 11.43 & & & & & \\
\hline $23_{0,23}-22_{1,22} \mathrm{E}$ & 239654.45 & 11.99 & & & & & \\
\hline $23_{1,23}-22_{1,22} E$ & 239654.55 & 61.66 & \multirow{6}{*}{90} & & & & \\
\hline $23_{0,23}-22_{0,22} \mathrm{E}$ & 239654.63 & 61.66 & & & & & \\
\hline $23_{1,23}-22_{0,22} E$ & 239654.72 & 11.99 & & 23965407 & 401 & 103 & 291 \\
\hline $23_{0,23}-22_{1,22} \mathrm{~A}$ & 239655.30 & 11.98 & & & & & \\
\hline $23_{1,23}-22_{1,22} \mathrm{~A}$ & 239655.39 & 61.67 & & & & & \\
\hline $23_{0,23}-22_{0,22} \mathrm{~A}$ & 239655.47 & 61.67 & & & & & \\
\hline $23_{1,23}-22_{0,22} \mathrm{~A}$ & 239655.57 & 11.98 & & & & & \\
\hline $24_{0,24}-23_{1,23} \mathrm{E}$ & 249859.75 & 12.54 & & & & & \\
\hline $24_{1,24}-23_{1,23} E$ & 249859.79 & 64.37 & & & & & \\
\hline $24_{0,24}-23_{0,23} \mathrm{E}$ & 249859.84 & 64.37 & & & & & \\
\hline $24_{1,24}-23_{0,23} E$ & 249859.89 & 12.54 & 98 & 24986081 & 107 & 68 & 303 \\
\hline $24_{0,24}-23_{1,23} \mathrm{~A}$ & 249860.54 & 12.54 & 90 & & & & \\
\hline $24_{1,24}-23_{1,23} \mathrm{~A}$ & 249860.59 & 64.38 & & & & & \\
\hline $24_{0,24}-23_{0,23} \mathrm{~A}$ & 249860.63 & 64.38 & & & & & \\
\hline $24_{1,24}-23_{0,23} \mathrm{~A}$ & 249860.68 & 12.54 & & & & & \\
\hline
\end{tabular}


Table 1. continued.

\begin{tabular}{|c|c|c|c|c|c|c|c|}
\hline $\begin{array}{c}\text { Trans. } \\
J_{K_{\mathrm{a}}^{\prime} K_{\mathrm{c}}^{\prime}}^{\prime}-J_{K_{\mathrm{a}}^{\prime \prime} K_{\mathrm{c}}^{\prime \prime}}^{\prime \prime}\end{array}$ & $\begin{array}{l}\text { Pred. } \\
\text { freq. } \\
(\mathrm{MHz})\end{array}$ & $\begin{array}{l}\mathrm{S} \mu^{2} \\
\left(\mathrm{D}^{2}\right)\end{array}$ & $\begin{array}{c}E_{\text {up }} \\
\left(\mathrm{cm}^{-1}\right)\end{array}$ & $\begin{array}{l}\text { Obs. } \\
\text { freq. }{ }^{a} \\
(\mathrm{MHz})\end{array}$ & $\begin{array}{c}\text { Obs. } \\
\int T_{\mathrm{mb}} \delta v \\
\left(\mathrm{mK} \mathrm{km} \mathrm{s}^{-1}\right)^{b}\end{array}$ & $\begin{array}{l}\text { Uncertertainty } \\
\text { Obs. } \int T_{\mathrm{mb}} \delta v \\
\left(\mathrm{mK} \mathrm{km} \mathrm{s}^{-1}\right)^{c}\end{array}$ & $\begin{array}{c}\text { Pred. } \\
\int T_{\mathrm{mb}} \delta v \\
\left(\mathrm{mK} \mathrm{km} \mathrm{s}^{-1}\right)^{d}\end{array}$ \\
\hline $25_{0,25}-24_{1,24} \mathrm{E}$ & 260063.90 & 13.10 & \multirow{8}{*}{106} & \multirow{8}{*}{260064.61} & \multirow{8}{*}{574} & \multirow{8}{*}{61} & \multirow{8}{*}{308} \\
\hline $25_{1,25}-24_{1,24} E$ & 260063.92 & 67.07 & & & & & \\
\hline $25_{0,25}-24_{0,24} \mathrm{E}$ & 260063.94 & 67.07 & & & & & \\
\hline $25_{1,25}-24_{0,24} \mathrm{E}$ & 260063.97 & 13.10 & & & & & \\
\hline $25_{0,25}-24_{1,24} \mathrm{~A}$ & 260064.63 & 13.10 & & & & & \\
\hline $25_{1,25}-24_{1,24} \mathrm{~A}$ & 260064.66 & 67.09 & & & & & \\
\hline $25_{0,25}-24_{0,24} \mathrm{~A}$ & 260064.68 & 67.09 & & & & & \\
\hline $25_{1,25}-24_{0,24} \mathrm{~A}$ & 260064.71 & 13.10 & & & & & \\
\hline $26_{0,26}-25_{0,25} \mathrm{E}$ & 270266.86 & 13.65 & \multirow{8}{*}{115} & \multirow{8}{*}{$270267.24^{*}$} & \multirow{8}{*}{-} & \multirow{8}{*}{$15 \mathrm{mK}$} & \multirow{8}{*}{363} \\
\hline $26_{1,26}-25_{1,25} \mathrm{E}$ & 270266.88 & 69.79 & & & & & \\
\hline $26_{0,26}-25_{1,25} \mathrm{E}$ & 270266.89 & 69.79 & & & & & \\
\hline $26_{1,26}-25_{0,25} \mathrm{E}$ & 270266.90 & 13.65 & & & & & \\
\hline $26_{0,26}-25_{1,25} \mathrm{~A}$ & 270267.55 & 13.64 & & & & & \\
\hline $26_{0,26}-25_{0,25} \mathrm{~A}$ & 270267.56 & 69.79 & & & & & \\
\hline $26_{1,26}-25_{1,25} \mathrm{~A}$ & 270267.57 & 69.79 & & & & & \\
\hline $26_{1,26}-25_{0,25} \mathrm{~A}$ & 270267.58 & 13.64 & & & & & \\
\hline
\end{tabular}

Notes. The lines with an asterisk are blended with other species, see text. ${ }^{(a)}$ Observed (centroid) frequencies assuming that the radial velocities relative to LSR are $3.9 \mathrm{~km} \mathrm{~s}^{-1}$, it does not coincide with the predicted frequency because it does not take into account blending with transitions of other species. ${ }^{(b)}$ Observed integrated intensity in main-beam temperature scale. Because of the high spectral confusion level it was not calculated for the line at $270.267 \mathrm{GHz}{ }^{(c)}$ Uncertainty on the observed integrated intensity in main-beam temperature scale calculated as follow: $\sigma=(1+\alpha) \times$ rms $\times \delta v \times \sqrt{N}, N$ being the number of channels over which the integrated intensities are computed, $\delta \mathrm{v}$ the channel width and $\alpha$ the uncertainty on the calibration of the observations ( $\alpha=17 \%$ in the 198-265 GHz spectral range, see Caux et al. 2010). For the line at $270.267 \mathrm{GHz}$, the rms of the observed spectrum in main-beam temperature scale is given. ${ }^{(d)}$ Integrated intensity of the modelled lines.

The line at $209.033 \mathrm{GHz}$ is partially blended with a line from $\mathrm{HCOOCH}_{3}$ (A species, 17 $11,6-16_{11,5}, 209.030 \mathrm{GHz}$ ). Nonetheless, the observations are reproduced well with a model including both the normal and deuterated species (Fig. 2). To model the $\mathrm{HCOOCH}_{3}$ emission, we used the same twocomponent LTE model as for the deuterated species. Tens of $\mathrm{HCOOCH}_{3}$ lines are present in the spectral survey. They were modelled with an excitation temperature of $100 \mathrm{~K}$ and $\mathrm{HCOOCH}_{3}$ abundances of $N_{\mathrm{A}, \mathrm{HCOOCH} 3} \sim 1 \times 10^{16} \mathrm{~cm}^{-2}$ and $N_{\text {B,HCOOCH3 }} \sim 9 \times 10^{15} \mathrm{~cm}^{-2}$. The detailed analysis of this species will be published elsewhere, however these values are consistent with those of Bottinelli et al. (2004) within the uncertainties. An excess of emission on the high-frequency side of the $\mathrm{HCOOCH}_{3}$ lines at 209.023, 209.033, and 209.044 GHz (although for the latter the line wing is within the noise; Fig. 2, panel 2) may be seen in the observed spectrum. Since this excess of emission is attributed to $\mathrm{DCOOCH}_{3}$ in the $209.033 \mathrm{GHz}$ line it is important to assess that it is not related to a $\mathrm{HCOOCH}_{3}$ line wing. This was checked on the numerous spectral lines assigned to $\mathrm{HCOOCH}_{3}$ in the spectral survey. It appears that they do not show any systematic emission excess, as can be seen in the panel (4) of Fig. 2, which shows two methyl formate lines at 229.405 and $229.420 \mathrm{GHz}$ that do no present such a feature.

The line at $219.242 \mathrm{GHz}$ is partially blended with a strong acetone $\left(\mathrm{CH}_{3} \mathrm{COCH}_{3}\right)$ line at $219.242 \mathrm{GHz}\left(21_{1,19}-20_{2,19}\right)$. Acetone contributes to about two thirds of the intensity of the observed line $\left(N_{\mathrm{CH} 3 \mathrm{COCH} 3} \sim 5 \times 10^{15} \mathrm{~cm}^{-2}, T_{\mathrm{ex}}=100 \mathrm{~K}, \theta_{\mathrm{s}}=1^{\prime \prime} .5\right.$, $V_{\mathrm{lsr}}=2.7 \mathrm{~km} \mathrm{~s}^{-1}$, and $\Delta v=4 \mathrm{~km} \mathrm{~s}^{-1}$ ) while $\mathrm{DCOOCH}_{3}$ contributes to one third (Fig. 2, panels 3a and 3b). It should be noted that the intensity of the two acetone lines at $219.220 \mathrm{GHz}$ and $219.265 \mathrm{GHz}$ (Fig. 2, panel 3b) are partially reproduced. We carefully checked that the adopted acetone model does not underestimate the abundance of acetone. It is impossible to account for the whole line-integrated intensities of these three lines without strongly contradicting other strong acetone lines in the survey. Typically, the intensity of the strongest acetone lines is overestimated by a factor 2 or 3 (depending on the parameters of the model) when the intensity of the 219.220, 219.242, and 219.265 GHz lines are correctly reproduced. Furthermore, the acetone lines are optically thin, independent of the model parameters. The optical depth of the strongest acetone lines do not exceed $7 \times 10^{-2}$, we thus do not expect any opacity problem that could affect the line intensities. The detailed analysis of acetone in the survey is postponed to a future paper, so we conclude that these two lines are blended with transitions of other species, although we have yet not identified which species, and the $219.242 \mathrm{GHz}$ band cannot be due to only acetone and is consistent with additional emission from $\mathrm{DCOOCH}_{3}$.

The transitions at 229.446, 239.654, 249. 860, and $260.064 \mathrm{GHz}$ do not appear to be blended. The observed line at $249.860 \mathrm{GHz}$ is narrower than the modelled line. However, we have checked that it is not an instrumental artefact or a spike, and it is probably caused by the low signal-to-noise ratio $(S / N \sim$ 1.6). The spectral range around the line at $270.26 \mathrm{GHz}$ is characterised by strong spectral confusion, however, although it is contaminated by nearby lines from other (unknown) molecules, the line at $270.267 \mathrm{GHz}$ is reproduced by the $\mathrm{DCOOCH}_{3}$ emission model reasonably well.

Thus, among the eight lines in the IRAM spectral range assigned to $\mathrm{DCOOCH}_{3}$, four lines are not blended with other 

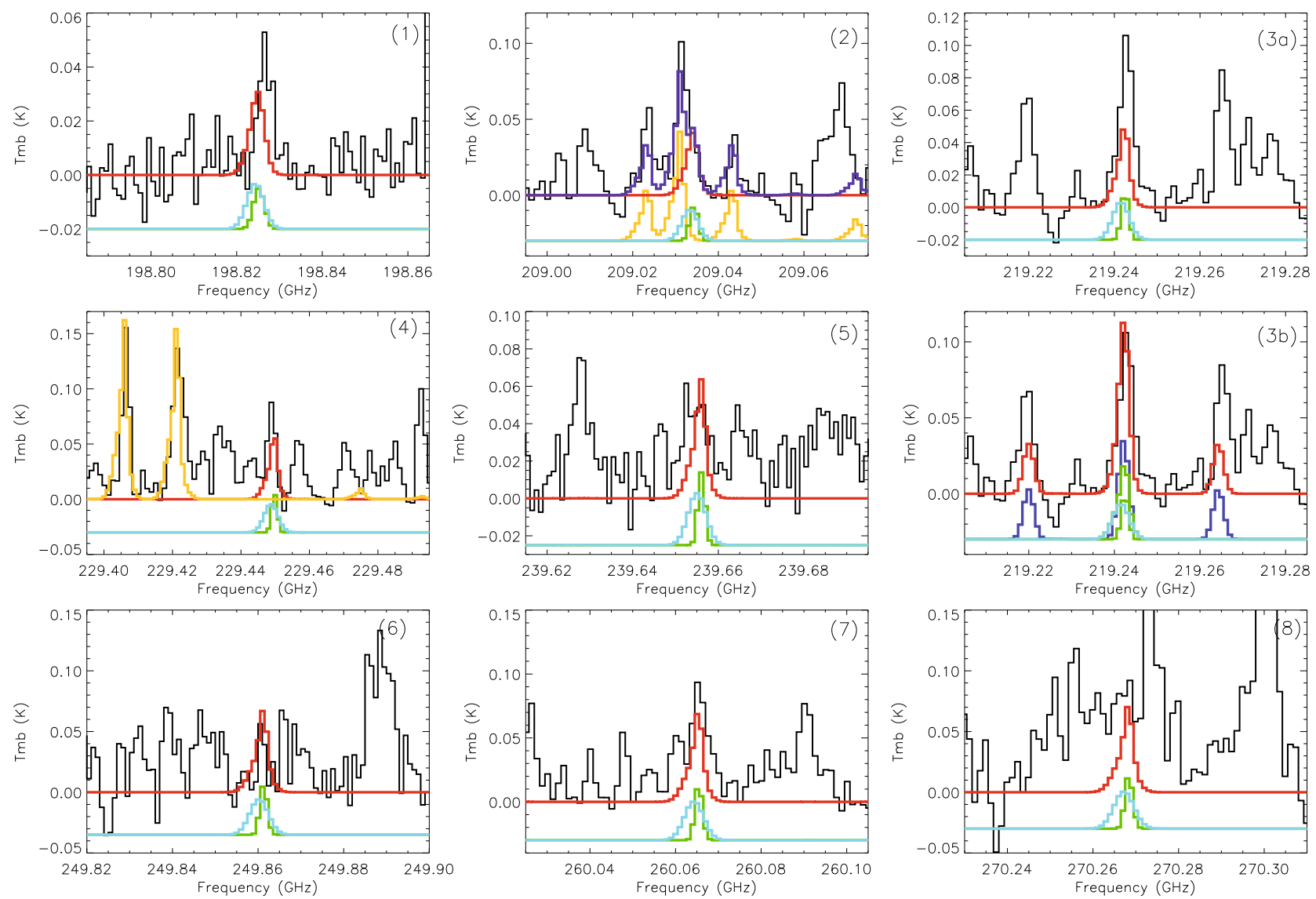

Fig. 2. Observed lines assigned to $\mathrm{DCOOCH}_{3}$ in IRAS 16293 compared with a two-components LTE emission model of $\mathrm{DCOOCH}_{3}$. Black line: observed IRAM spectral lines. Red line: sum of the emission from region A (light blue line) modelled with $\theta_{s}=2^{\prime \prime}, T_{\mathrm{ex}}=100 \mathrm{~K}, V_{\mathrm{lsr}}=3.9 \mathrm{~km} \mathrm{~s}{ }^{-1}$ and $\Delta v=6 \mathrm{~km} \mathrm{~s}^{-1}, N_{\mathrm{A}, \mathrm{DCOOCH}} \sim 6 \times 10^{14} \mathrm{~cm}^{-2}$, and region B (green line) modelled with $\theta_{\mathrm{s}}=1.5^{\prime \prime}, T_{\mathrm{ex}}=100 \mathrm{~K}, V_{\mathrm{lsr}}=2.7 \mathrm{~km} \mathrm{~s}{ }^{-1}$ and $\Delta v=2 \mathrm{~km} \mathrm{~s}^{-1}, N_{\mathrm{B}, \mathrm{DCOOCH}} \sim 6 \times 10^{14} \mathrm{~cm}^{-2}$. The yellow line in panels 2) and 4) is the emission from $\mathrm{HCOOCH}_{3}$ modelled with the same two components A and B (see text). Panels 3a) and 3b) both show the line at $219.242 \mathrm{GHz}$ : 3a) emission from the two components of $\mathrm{DCOOCH}_{3}$ and their sum, 3b) DCOOCH 3 emission (green and light blue lines) and the $\mathrm{CH}_{3} \mathrm{COCH}_{3}$ emission (dark blue line, see text), the sum of these emissions is shown in red. For the sake of clarity the light blue, dark blue, and the green lines have been shifted downward.

species $(229.448,239.654,249.860,260.064 \mathrm{GHz})$, two lines blended with known species $\left(\mathrm{HCOOCH}_{3}\right.$ at $209.031 \mathrm{GHz}$ and $\mathrm{CH}_{3} \mathrm{COCH}_{3}$ at $219.242 \mathrm{GHz}$ ). They are reproduced well by the model, and $\mathrm{DCOOCH}_{3}$ contributes about $30-35 \%$ to their intensity, independent of the adopted model used for acetone and methyl formate. The line at $270.267 \mathrm{GHz}$ is heavily blended with unknown species, but the emission model agrees with the observations. The emission model is compatible with the observations at $198.824 \mathrm{GHz}$, however because this observation suffers from calibration uncertainty, it is impossible to conclude about the detection of this line.

For $\mathrm{DCOOCH}_{3}$, a reasonable agreement with the observations is found for a column density of $N_{\mathrm{A}, \mathrm{DCOOCH} 3}=$ $N_{\mathrm{B}, \mathrm{DCOOCH} 3} \sim 6 \times 10^{14} \mathrm{~cm}^{-2}$. High angular resolution observations are needed not only to confirm the adopted two-component model for $\mathrm{DCOOCH}_{3}$, but also to clarify the situation regarding $\mathrm{HCOOCH}_{3}$. Indeed, in their interferometric study of this species, Bottinelli et al. (2004) measured an apparent difference between the $V_{\mathrm{lsr}}$ and line widths of the two hot corinos, namely $V_{\mathrm{lsr}}=3.9 \mathrm{~km} \mathrm{~s}^{-1}$ and $\Delta v=6 \mathrm{~km} \mathrm{~s}^{-1}$ for source A, and $V_{\mathrm{lsr}}=2.7 \mathrm{~km} \mathrm{~s}^{-1}$ and $\Delta v=2 \mathrm{~km} \mathrm{~s}^{-1}$ for source B. However, the authors were unable to conclude whether the values for the $V_{\text {lsr }}$ and line width in source B were real or caused by the distortion of optically thick $\mathrm{HCOOCH}_{3}$ lines in source $\mathrm{B}$, in which case the blue wing of the (strongly self-absorbed) broad line normally centred at $3.9 \mathrm{~km} \mathrm{~s}^{-1}$ could be mistaken for a narrow line centered at $2.7 \mathrm{~km} \mathrm{~s}^{-1}$. The $\mathrm{DCOOCH}_{3}$ lines are optically thin. Therefore, if the two-component model used in this study is confirmed by higher angular resolution observations, it would imply that the value of $V_{\mathrm{lsr}}$ and of the line width are indeed different in the two hot corinos in IRAS 16293.

Using the column density previously derived for $\mathrm{HCOOCH}_{3}$ and $\mathrm{DCOOCH}_{3}$, the degree of deuteration of methyl formate in IRAS 16293 is estimated to $\sim 15 \%$. This degree of deuteration is similar in sources A and B but dedicated high spatial resolution observations are needed to confirm this $\left(\mathrm{HCOOCH}_{3}\right.$ could be optically thick in the core $\mathrm{B}$ ). Although this is a rough estimate, the deuterium enrichment of methyl formate, greater than the one expected from deuterium cosmic abundance, is compatible with other singly deuterated species in this source such as $\mathrm{HDCO}$ or $\mathrm{CH}_{2} \mathrm{DOH}$ that have a deuterium enrichment of $\sim 15 \%$ and $37 \%$, respectively (Parise et al. 2006).

\section{Discussion}

It has been established that methyl formate cannot be formed exclusively by gas-phase reactions (Horn et al. 2004) and that solid state reactions necessarily play a role in its formation. Methyl formate may form in the icy mantle of dust grains via the reaction between the metoxy radical, $\mathrm{CH}_{3} \mathrm{O}$, with the formyl radical, $\mathrm{HCO}$. Both $\mathrm{CH}_{3} \mathrm{O}$ and $\mathrm{HCO}$ are synthesised in the grain mantles either by hydrogenation of $\mathrm{H}_{2} \mathrm{CO}$ and $\mathrm{CO}$, respectively 
(Garrod \& Herbst 2006; Watanabe \& Kouchi 2008) or via UV photodissociation of methanol, which produces $\mathrm{CH}_{3} \mathrm{O}$ and an $\mathrm{H}$ atom that reacts with $\mathrm{CO}$ to produce $\mathrm{HCO}$ (Bennett \& Kaiser 2007). As for methyl formate, it is likely that deuterated methyl formate formation also requires grain chemistry. We discuss below two formation pathways, implying solid state reactions, that may lead to $\mathrm{DCOOCH}_{3}$.

The first one is similar to the formation of $\mathrm{HCOOCH}_{3}$. In that scenario, $\mathrm{DCOOCH}_{3}$ is formed directly via the reaction: $\mathrm{DCO}+\mathrm{CH}_{3} \mathrm{O} \rightarrow \mathrm{DCOOCH}_{3}$ (1). At low temperature and in the absence of energetic processes, DCO could be produced by deuteration reaction of $\mathrm{CO}$ with gas phase deuterium atom accreted onto the grain, while $\mathrm{CH}_{3} \mathrm{O}$ results from the hydrogenation of $\mathrm{H}_{2} \mathrm{CO}$. However, experiments from Hidaka et al. (2007) have shown that the deuteration of $\mathrm{CO}$ ice is about 10 times less than the hydrogenation rate because there is no tunnelling effect for deuteration. The authors thus conclude that the deuteration of $\mathrm{CO}$ may not be the first step in producing deuterated formaldehyde or methanol in molecular clouds. This may also be the case for the formation of deuterated methyl formate.

Energetic processes in ices, such as UV photolysis or cosmic rays bombardment of ices, may be more efficient at producing $\mathrm{DCOOCH}_{3}$ via reaction (1). Following the formation of methyl formate reported by Bennett \& Kaiser (2007), DCO could be formed from the reaction of $\mathrm{CO}$ with a deuterium atom produced by the UV photodissociation of deuterated methanol. The energy given by the UV photons should help overcome the activation energy of the reaction $\mathrm{CO}+\mathrm{D} \rightarrow \mathrm{DCO}$. Among the deuterated isotopologues of methanol, $\mathrm{CH}_{3} \mathrm{OD}$ seems to be the best candidate for the UV photodissociation, since if the UV photons break the $\mathrm{O}-\mathrm{D}$ bond of $\mathrm{CH}_{3} \mathrm{OD}, \mathrm{CH}_{3} \mathrm{O}$ and a $\mathrm{D}$ atom are easily produced. This is not the case for any other deuterated methanol species, for which the simultaneous production of $\mathrm{CH}_{3} \mathrm{O}$ and $\mathrm{D}$ would require intermediate reactions.

A second route to forming deuterated methyl formate is the $\mathrm{H} / \mathrm{D}$ substitution in solid $\mathrm{HCOOCH}_{3}$ during the warm-up of the ice mantles. This scenario is proposed for the formation of deuterated formaldehyde and participates in the formation of deuterated methanol species (Hidaka et al. 2009; Ratajczak et al. 2009). It may also be efficient at producing deuterated methyl formate. Once again, the H/D substitution could also be driven by energetic processes such as UV photolysis or cosmic-ray irradiation of the ice mantles (Weber et al. 2009). In their study of methane/deuterated-water ice, Weber et al. (2009) conclude that almost all organic species should undergo H/D substitution with the matrix in water ices exposed to UV radiation.

Isotopic substitutions are routinely used in laboratory experiments to disentangle the different chemical pathways leading to the formation of molecules. From that point of view, the formation of deuterated methyl formate is an interesting issue that could improve our understanding of the chemistry of these species. To our knowledge, experimental and theoretical works on the deuteration of methyl formate and other complex organic molecules do not exist yet. They are clearly needed to distinguish between the above scenarii. The astronomical detection of other deuterated isotopologues of methyl formate should also put some constraints on the different formation pathways.

\section{Conclusion}

We have tentatively detected the singly-deuterated isotopologue of methyl formate, $\mathrm{DCOOCH}_{3}$, in the protostar IRAS16293. We assigned eight observed lines to $\mathrm{DCOOCH}_{3}$ transitions. Four lines are not blended with other species (229.448, 239.654,
249.860, 260.064 GHz), and three are (209.031, 219.242, $270.267 \mathrm{GHz}$ ). Among the last, the first two lines are blended with $\mathrm{HCOOCH}_{3}$ and $\mathrm{CH}_{3} \mathrm{COCH}_{3}$, respectively. They are reproduced well by an emission model at LTE. We were not able to identify the species responsible for the blending of the $270.267 \mathrm{GHz}$ line, however the LTE model is compatible with the observations and does not contradict the tentative identification of $\mathrm{DCOOCH}_{3}$ in IRAS 16293. The LTE emission model is compatible with the last observed line at $198.824 \mathrm{GHz}$, but we cannot conclude anything about its detection because of calibration uncertainty. From basic modelling in LTE, we estimate the abundance of deuterated methyl formate to be $N_{\mathrm{A}+\mathrm{B}, \mathrm{DCOOCH} 3} \sim$ $1.2 \times 10^{15} \mathrm{~cm}^{-2}$. This leads to a $\mathrm{DCOOCH}_{3} / \mathrm{HCOOCH}_{3}$ ratio of $\sim 15 \%$, consistent with the deuteration fractionation of other singly deuterated species in this source.

Additional observations with better spectral resolution and higher sensitivity are needed for several reasons. First, considering the low spectral resolution and the relatively low signalto-noise ratio of the lines assigned to $\mathrm{DCOOCH}_{3}$, such new observations would strengthen this tentative detection. Second, it would allow detection of additional lines among the hundreds of weaker lines from $\mathrm{DCOOCH}_{3}$ that are present in the spectral survey. Last, it would be an opportunity to search for other deuterated isotopologues of methyl formate. High angular resolution observations would also be helpful for investigating the existence of two emission sources for $\mathrm{DCOOCH}_{3}$, as observed for $\mathrm{HCOOCH}_{3}$ and for concluding about the reality of the physical differences between the two cores A and B. Such observations are also needed to properly estimate the methyl formate deuteration and, together with new experimental and theoretical work, to understand the deuteration mechanisms of complex organic molecules.

Acknowledgements. The authors would like to thank Laurent Margulès for providing us with the $\mathrm{DCOOCH}_{3}$ spectral data prior to publication. We thank the IRAM staff for help provided during the observations. Support by the French National Agency (ANR-02-BLAN-0225-01) is acknowledged.

\section{References}

Bacmann, A., Lefloch, B., Ceccarelli, C., et al. 2003, ApJ, 585, L55 Bennett, C. J., \& Kaiser, R. I. 2007, ApJ, 661, 899

Bisschop, S. E., Jørgensen, J. K., Bourke, T. L., Bottinelli, S., \& van Dishoeck, E. F. 2008, A\&A, 488, 959

Bottinelli, S., Ceccarelli, C., Neri, R., et al. 2004, ApJ, 617, L69

Caux, E., Kahane, C., Castets, A., et al. 2010, A\&A, submitted

Cazaux, S., Tielens, A. G. G. M., Ceccarelli, C., et al. 2003, ApJ, 593, L51

Ceccarelli, C., Loinard, L., Castets, A., et al. 2001, A\&A, 372, 998

Garrod, R. T., \& Herbst, E. 2006, A\&A, 457, 927

Hidaka, H., Kouchi, A., \& Watanabe, N. 2007, J. Chem. Phys., 126, 204707

Hidaka, H., Watanabe, M., Kouchi, A., \& Watanabe, N. 2009, ApJ, 702, 291

Horn, A., Møllendal, H., Sekiguchi, O., et al. 2004, ApJ, 611, 605

Jacq, T., Walmsley, C. M., Mauersberger, R., et al. 1993, A\&A, 271, 276

Leurini, S., Rolffs, R., Thorwirth, S., et al. 2006, A\&A, 454, L47

Margulès, L., Huet, T. R., Demaison, J., et al. 2010, ApJ, 714, 1120

Parise, B., Castets, A., Herbst, E., et al. 2004, A\&A, 416, 159

Parise, B., Ceccarelli, C., Tielens, A. G. G. M., et al. 2006, A\&A, 453, 949

Pety, J., Goicoechea, J. R., Hily-Blant, P., Gerin, M., \& Teyssier, D. 2007, A\&A, 464, L41

Pillai, T., Wyrowski, F., Hatchell, J., Gibb, A. G., \& Thompson, M. A. 2007, A\&A, 467, 207

Ratajczak, A., Quirico, E., Faure, A., Schmitt, B., \& Ceccarelli, C. 2009, A\&A, 496, L21

Roberts, H., Herbst, E., \& Millar, T. J. 2003, ApJ, 591, L41

Tielens, A. G. G. M. 1983, A\&A, 119, 177

Turner, B. E. 1998, ApJ, 501, 731

Watanabe, N., \& Kouchi, A. 2008, Prog. Surf. Sci., 83, 439

Weber, A. S., Hodyss, R., Johnson, P. V., Willacy, K., \& Kanik, I. 2009, ApJ, 703,1030 